Josef Roos

Prof. Dr. med. Gerd Nagel: «Integrative Medizin - Herausforderung für die Onkologie» Integrative Medizin: Es gibt weltweit viele medizinische Systeme und Schulen - aber welche ist die richtige Medizin? Die Antwort ist überraschend einfach: Die richtige Medizin ist die für den einzelnen Patienten richtige Medizin. Man hat sie ganzheitliche Medizin genannt, oder auch integrative Medizin. Integrative Medizin, weil es vor allem um die Integration von zwei unterschiedlichen Denkstilen in ein ganzheitliches Handlungsmuster - bei uns: dem eher naturwissenschaftlich ausgerichteten Denkstil der Schulmedizin und dem eher naturheilkundlich ausgerichteten Denkstil von Patienten - geht.

Das Zwei-Ärzte-Modell von Krebspatienten: Die meisten Krebspatienten sind davon überzeugt, dass es zur Bewältigung der Krebserkrankung zwei Ärzte braucht - den äusseren und den inneren Heiler. Dieses integrative «Modell der zwei Ärzte» geht zurück auf den Arzt Hippokrates (460-377 v. Chr.), der den natürlichen Heilkräften, der vis medicatrix naturae, sehr grosse Bedeutung einräumte. Im System der schulmedizinischen Onkologie des 20. Jahrhunderts fehlt das Konzept des inneren Arztes. Moderne, kompetente Patienten fordern aber immer deutlicher unser Eingehen auf patienteneigene Ressourcen. Aber wie soll das der Onkologe bewerkstelligen, ohne sich dem Vorwurf der «Unwissenschaftlichkeit» auszusetzen?

Komplementärmedizin und komplementäre Wirklichkeiten: So wie sich pathotropes und salutotropes Han-

\title{
2. Aeskulap-Symposium in der Aeskulap-Klinik
}

\author{
Symposium zum Thema «Integrative Onkologie»
}

Am 16. September 2010 lud die Aeskulap-Klinik in Brunnen zum 2. AeskulapForum ein. Das diesjährige Symposium widmete sich der Integrativen Onkologie, einem Thema, welches in der Schweiz eine wachsende Bedeutung erfährt. Ziel war es, schulmedizinische als auch komplementärmedizinische Sichtweisen in der Behandlung onkologischer Erkrankungen sinnvoll mit dem Patienten zu diskutieren sowie Nützliches und Erwiesenes ohne ideologische Barrieren zusammenzuführen. Das Symposium, an welchem elf hochkarätige Referenten auftraten, zeigte in diesem Zusammenhang Ansätze auf, die schon heute begleitend zu klassischen Massnahmen erfolgen. Lesen Sie nachstehend die Zusammenfassungen der Symposiumsbeiträge.

deln komplementär zueinander verhalten, handelt es sich bei den unterschiedlichen Wahrnehmungen ein und derselben medizinischen Situation durch den Arzt einerseits und den Patienten andererseits um das $\mathrm{Zu}$ sammentreffen sog. komplementärer Wirklichkeiten. Die Komplementärmedizin rechtfertigt sich nicht aus der ärztlichen, sondern der dazu komplementären Wirklichkeit des Patienten. Anerkennt man das Recht des Patienten, sich selbst, in eigenem Ermessen, helfen $\mathrm{zu}$ wollen, stellt sich die Frage nach den Pflichten des Arztes, wenn der Patient in eigener Sache zur Selbsthilfe greifen will.

Evidenzbasierte Komplementärmedizin: Aus dem Recht des Patienten nach selbstverantwortetem Handeln in eigener Sache und der Pflicht des Arztes, den Patienten vor Schaden zu bewahren, ergibt sich das Denkmodell der evidenzbasierten Komplementärmedizin. Da der Nutzen seiner Eigenaktivitäten nur vom Patienten selbst beurteilt werden kann (subjektive Evidenz), liegt das ärztliche Augenmerk auf Aspekten der Sicherheit der Kom- plementärmedizin. In Bezug auf den Sicherheitsaspekt der Komplementärmedizin wurden in den letzten Jahren grosse Fortschritte erzielt. Bestes Beispiel ist die Mikronährstoffmedizin.

\section{Dr. med. Marc Schlaeppi: «Warum ganzheitliche Beratung in der Onkologie?»}

Wenn der Mensch sich als soziales Wesen, bestehend aus Geist, Seele und Körper, versteht, sollte die Medizin dieser Tatsache Rechnung tragen. Engel hatte 1977 in seinem LandmarkArtikel in Science von der Notwendigkeit einer biopsychosozialen Medizin geschrieben und einen ersten Paradigmenwechsel eingeleitet. Verschiedene «whole medical systems» in der Komplementärmedizin berücksichtigen typischerweise einen mehrdimensionalen Menschen. Eine wissenschaftliche Integrative Medizin, die Komplementärmedizin und konventionelle Medizin zusammenbringt, könnte einen Beitrag zu einer ganzheitlichen Betrachtung und Patientenberatung leisten.

\section{KARGER}

Fax +497614520714
(๑) 2010 S. Karger GmbH, Freiburg

Accessible online at: www.karger.com/szg
Dr. Josef Roos (Medienverantwortlicher)

Aeskulap-Klinik

Gersauerstrasse 8, 6440 Brunnen, Schweiz

Tel. +41 41 825-4747, Fax -4965

josef.roos@aeskulap.com 


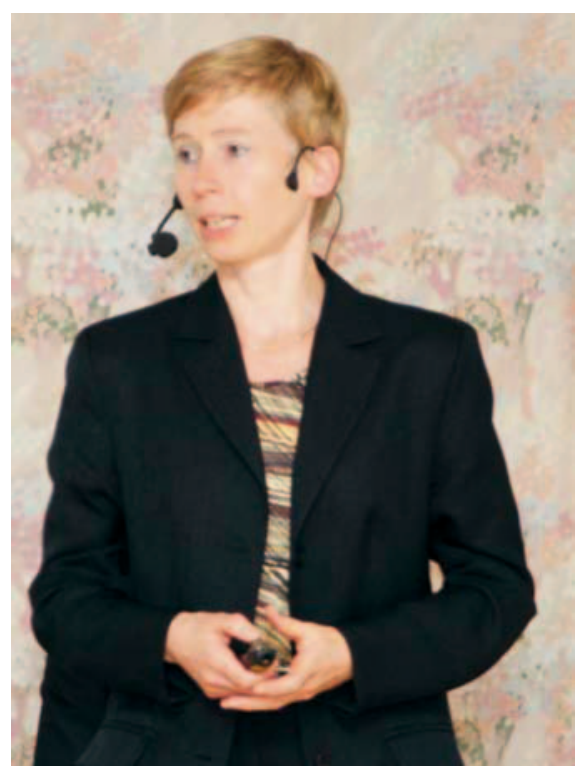

Dr. med. Jutta Hübner.

\section{Dr. med. Jutta Hübner:}

«Naturheilkundliche Ansätze was geht zusammen, was nicht?»

Seit der Publikation zur Wirkungsabschwächung von Irinotecan durch Johanniskraut richtet sich die Aufmerksamkeit insbesondere auf Wechselwirkungen über den Metabolismus von Medikamenten durch Cytochrom P 450 3A4. Dabei gibt es viele weitere Interaktionsmöglichkeiten über weitere Cytochrom-Enzyme, wie z.B. 2D6, oder auch über Rezeptoren und die intrazellulären Targets moderner antitumoraler Medikamente.

Allein die Daten zu den Vitaminen füllen Bände und bleiben trotzdem widersprüchlich. Vitamin C schwächt in vitro die Zytotoxizität von Zytostatika ab, und zwar nicht nur durch eine antioxidative Wirkung, sondern indem die Depolarisation der Mitochondrienmembran inhibiert wird. Umgekehrt scheint Vitamin C die Wirkung anderer Chemotherapeutika an Tumorzellen zu verstärken. Auch für Vitamin D3 liegen Daten für eine synergistische Wirkung mit verschiedenen Zytostatika vor. Selen hat bisher, obwohl es als Antioxidanz eingestuft wird, in den experimentellen Untersuchungen durchgehend eine Wirkungsverstärkung von Zytostati- ka sowohl z.B. der Taxane als auch der Antracycline gezeigt. Nicht alle Interaktionen müssen also negativ sein. Im Bereich der sekundären Pflanzenstoffe gibt es interessante Kandidaten, die in Studien weiterentwickelt werden können, um die Wirkung einer Chemotherapie zu verstärken. Hierzu gehören EGCG (Epigallocatechingallat), Curcumin und Resveratrol.

Eine häufigere Indikation ist die supportive Therapie mit komplementären Methoden. Es gibt Nebenwirkungen, für die die konventionelle Medizin keine Antworten hat. Hierzu gehören Fatigue und Polyneuropathie. Die komplementäre Onkologie weist mögliche Antworten auf, so z.B. zur Behandlung der Fatigue mit Carnitin oder Ginseng. Aber auch hierbei müssen im Rahmen eines individuellen Therapiekonzeptes mögliche Interaktionen sorgfältig abgeschätzt werden. Aus diesem Grund gehört die Beratung zur komplementären Onkologie in die Hand des erfahrenen Onkologen. Zunächst geht die Sicherheit unserer Patienten vor - d.h., wenn wir Bedenken bezüglich Interaktionen haben, so sollten wir abraten.

Für die Zukunft brauchen wir die Einbeziehung von komplementären Fragestellungen in klinische Studien und die gezielte Weiterentwicklung von In-vitro-Daten über tierexperimentelle Ansätze in die klinische Forschung.

\section{Helmut Knorr: \\ «Ganzheitliche Ansätze \\ in der Palliativmedizin»}

Kennzeichen der Palliativmedizin ist ein im höchsten Masse patientenzentriertes Vorgehen mit dem erklärten Ziel, die letzte Lebensphase trotz krankheitsbedingter Einschränkungen möglichst angenehm zu gestalten (Lebensqualitätserhaltung). Bei Berücksichtigung der jeweils unterschiedlichen individuell gegebenen physischen, psychischen und spirituellen Bedürfnisse des einzelnen Menschen erfordert dies ein individuelles, am Einzelnen orientiertes Vorgehen und eine umfassende Betrachtung dieser Bedürfnisse - ein ganzheitliches Vorgehen.

In unserer Klinik nutzen wir neben den etablierten Verfahren der Schulmedizin Methoden der Komplementärmedizin, um diesem Anspruch gerecht $\mathrm{zu}$ werden. Theoretische wie auch praktische Aspekte einer ganzheitlichen Palliativmedizin werden berücksichtigt.

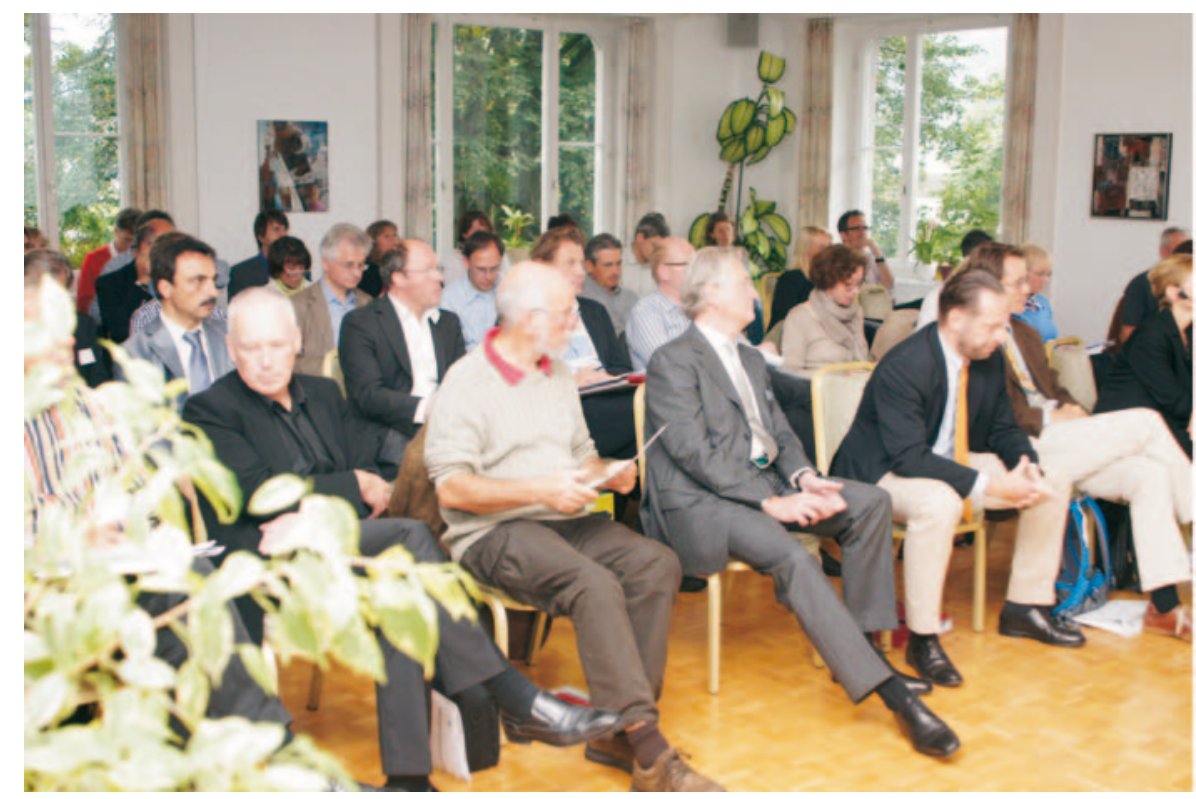


Dr. med. Peter Holzhauer:

«Der Einsatz von Vitaminen

und Spurenelementen in der

Prophylaxe von Nebenwirkungen

der Tumortherapie»

Zahlreiche Untersuchungen zum Einsatz komplementärer Behandlungsmassnahmen belegen, dass ein grosser Teil der an Krebs erkrankten PatientInnen sog. komplementäre oder ergänzende Therapien einsetzen. Auch die Motive für die Inanspruchnahme sind gut untersucht und unter anderem in dem Wunsch nach Eigenbeteiligung, Mitentscheidung und Autonomie und damit der Vorstellung einer Prognoseverbesserung verbunden. Ein weiterer wichtiger Grund für den Einsatz ausgewählter komplementärer Verfahren ist der Versuch, dadurch die verschiedenen Nebenwirkungen, die mit einer Tumortherapie, ob medikamentös oder nichtmedikamentös, einhergehen können, zu vermindern.

Der Einsatz von Mikronährstoffen wird in diesem Kontext sehr kontrovers diskutiert. Kritiker solcher begleitender Massnahmen befürchten eine Verminderung der Effektivität der konventionellen Tumortherapie durch Interaktionen. Die Rationale für den Einsatz von Mikronährstoffen als supportive und komplementäre Massnahme liegt in der Vorstellung, eine defizitäre Versorgung von $\mathrm{Tu}$ morpatienten mit kritischen Mikronährstoffen, wie z.B. Selen, L-Carnitin und Vitamin D, zu kompensieren und damit auch einem erhöhten Bedarfbei dieser Patientengruppe gerecht $\mathrm{zu}$ werden. Für Selen und L-Carnitin wurden Mangelzustände durch verminderte Aufnahme, z.B. bei tumorbedingter Mangelernährung, oder iatrogen induzierte Mikronährstoffdefizite durch Interaktionen mit $\mathrm{Zy}$ tostatika wie Ifosfamid, Cisplatin oder Doxorubicin beschrieben.
Dr. med. Boris Müller-Hübenthal: «Einsatz von Mistelpräparaten in der Onkologie»

Mistelpräparate gehören $\mathrm{zu}$ den am häufigsten verordneten komplementären Therapieverfahren in der Onkologie in Mitteleuropa. Ihr Einsatz erfolgt unter der Vorstellung einer direkten antitumoralen Wirkung, zur Verbesserung der Lebensqualität und gleichzeitiger Abschwächung therapieassoziierter Toxizität. Mistel enthält Lektine (Lektin I, II und III; hochmolekulare Polypeptide), lösliche Triterpene (insbesondere Oleanolsäure), Terpenoide, Viscotoxine A2, A3 und B (niedermolekulare Polypeptide). Das Referat gab eine Übersicht über die Studienlage und einen Einblick in den praktischen klinischen Einsatz der Misteltherapie im Rahmen eines integrativen onkologischen Behandlungskonzepts.

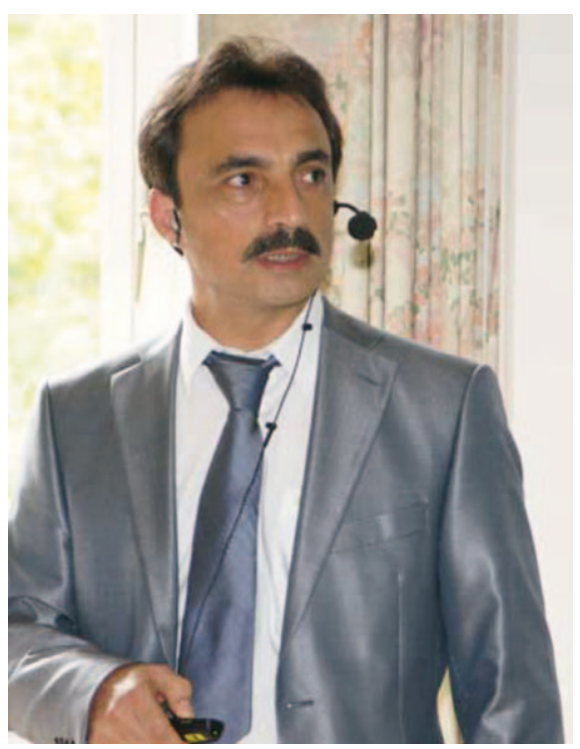

Dr. med. Hüseyin Sahinbas.

Dr. med. Hüseyin Sahinbas: "Stellenwert der Hyperthermie in der modernen Onkologie»

Hyperthermie, auch Thermotherapie genannt, ist eine Form einer multidisziplinären Krebsbehandlung, bei der das Körpergewebe erhöhten Temperaturen ausgesetzt wird, mit dem Ziel, Tumorgewebe zu zerstören und Syn- ergieeffekte mit Zytostatika, Strahlen und Antikörpern auszunutzen. Hyperthermie ist neben der Immuntherapie eine weitere Säule in der Krebstherapie, die komplementär zur Chemound Strahlentherapie eingesetzt werden kann.

Die Hyperthermie ist eine gut untersuchte und vielversprechende innovative Methode in der Krebstherapie. Die Ergebnisse experimenteller und klinischer Studien weisen darauf hin, dass Hyperthermie eine ideale komplementäre Behandlung und ein starker Sensibilisator für die Radiooder Chemotherapie ist. Neue technische Methoden und umfassende experimentelle und klinische Prüfungen haben die Effizienz dieser Methode demonstriert und rechtfertigen deren Anwendung in der Krebstherapie zum Vorteil der Patienten.

Je nach Lage, Grösse und Entität der Tumoren kommen verschiedene technische Methoden der Wärmeinduktion zur Anwendung.

Generelle Wirkmechanismen der Hyperthermie (in Abhängigkeit von der Temperatur):

1. Direkte Tumornekrose durch Hitzeeinwirkung.

2. Erhöhte Durchblutung im gesunden Gewebe führt zur Nährstoff- und Sauerstoffverarmung des Tumorgewebes.

3. Blutarmut im Tumor hat wiederum eine Nährstoff- und Sauerstoffverarmung des Tumorgewebes zur Folge.

4. Dies führt zur anaeroben Energiegewinnung über den Zuckerabbau zu einem sauren Milieu im Tumor (Apoptose durch anaerobe Stoffwechselinduktion).

5. Sensibilisierung der Strahlen- und Chemotherapie (synergistischer Effekt): Unter Hyperthermie werden einzelne Chemotherapeutika in ihrer Wirkungsweise bis zum Fünffachen gesteigert, ohne dass die Nebenwirkungen mit ansteigen.

6. Durchbrechung der Chemo- und Strahlenresistenz. 
7. Durch die Hyperthermie (wie auch bei Chemo- oder Strahlentherapie) wird die Expression von Stressproteinen (HSP) ausgelöst; dies verstärkt eine Oberflächenpräsentation des Tumors für das Immunsystem (Kenntlichmachung der Tumorzellen $=$ Erhöhung der Immunogenität der Tumorzellen $\rightarrow$ Tumorlyse durch T-Zellen).

8. Die Wärme aktiviert die ruhenden Zellen aus der G0-Phase in die G1- bzw. S-Phase und macht sie für die Chemo- oder Strahlentherapie zugängig.

9. Die Mikroembolisation der Krebsgefässe (angiogenetischer Block) konnte durch angiographische Untersuchungen nachgewiesen werden.

10. Schmerzreduktion.

11. Erhöhung der Lebensqualität (Karnofsky-Index).

Hyperthermie in Ergänzung zur Radiotherapie: Verschiedene Mechanismen sind für den supra-additiven Effekt der Hyperthermie in Kombination mit der Strahlentherapie verantwortlich. Hyperthermie kann ausserdem eine erhöhte Durchblutung bewirken, die zu einer Steigerung der Oxygenierung des Gewebes führt, was in einer erhöhten Radiosensitivität resultiert. Hyperthermie verstärkt ausserdem zytotoxische Strahleneffekte, insbesondere durch die Interferenz mit dem zellulären Reparatursystem. In-vivo-Studien haben gezeigt, dass der Effekt der Strahlentherapie durch die Hyperthermie um den Faktor 1,2 bis 5 verstärkt werden kann. Zusammenfassend ist die Hyperthermie wahrscheinlich der potenteste Radiosensibilisator, der zurzeit bekannt ist.

Hyperthermie in Ergänzung zur Chemotherapie: Die Wechselwirkungen von Hyperthermie und Zytostatika können unabhängig, additiv oder supra-additiv (synergistisch) sein. Die wichtigsten Mechanismen, die zu einem interaktiven Effekt mit Zyto- statika führen, sind unter anderem eine erhöhte intratumorale Arzneimittelkonzentration, die auf eine gesteigerte Durchblutung und erhöhte Permeabilität der Membran mit einer gesteigerten intrazellulären Aufnahme zurückzuführen ist, sowie ein gesteigerter intrazellulärer Arzneimittelmetabolismus und eine beschleunigte Reaktionsrate.

Synergieeffekte können aber auch durch Veränderungen im Gewebe und den veränderten Metabolismus von Zytostatika infolge von Wärme entstehen (z.B. 5-FU). Neuere tierexperimentelle Untersuchungen zeigen einen sehr engen zeitlichen Zusammenhang in der Reihenfolge der Applikation von Zytostatika und Hyperthermie, die für die Therapieplanung von grosser Bedeutung sind.

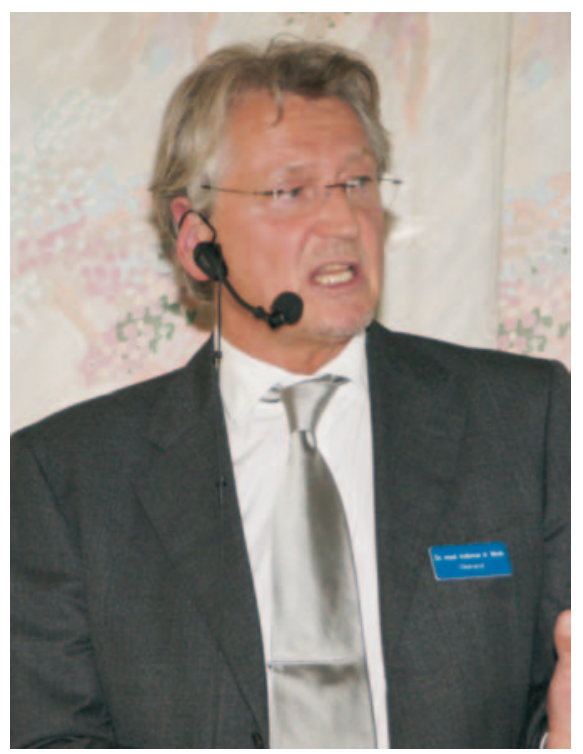

Dr. med. Volkmar A. Wirth

Dr. med. Volkmar Wirth: "Lokoregionale Tiefenhyperthermie - eigene Erfahrungen»

Seit März 2009 führt die Abteilung Onkologie die lokoregionale Tiefenhyperthermie in Form der Elektrohyperthermie mit dem Gerätetyp «Celsius TCS» der Fa. Celsius 42+ durch. Im Zeitraum von März 2009 bis Juli 2010 wurden insgesamt 900 Behandlungen an 79 Patienten durchgeführt. Die vorwiegend ambulant durchgeführten Therapien wurden meist mit simultan verabreichten Chemotherapien und/oder mit unseren komplementären Behandlungsverfahren kombiniert. Bis auf wenige Ausnahmen wurden die Therapien 2-mal/ Woche mit je 60 min Behandlungsdauer angewandt. Die durchschnittlichen Behandlungszyklen pro Patient umfassten jeweils 10-12 Hyperthermiesitzungen.

Es werden eine Zusammenschau der gewonnenen Erfahrungen, die Trends der Effektivität und deren mögliche Verstärker-Wirkung auf die üblichen onkologischen Standardverfahren bei unterschiedlichsten Entitäten herausgearbeitet. Anhand einzelner exemplarischer Fallbeispiele werden besonders beachtenswerte Verläufe dokumentiert.

Das kaum nennenswerte Nebenwirkungsprofil und die minimale Kontraindikationspalette entsprachen der international veröffentlichten $\mathrm{Da}$ tenlage und bekräftigen in Kombination mit den oben erwähnten Trends den Wunsch nach vermehrter Studientätigkeit in dieser hoffnungsvollen, aus unserer Sicht noch zu wenig beachteten Disziplin.

Prof. Dr. med. Marcus Schuermann: «Östrogenbasierte Phytotherapie beim Prostatakrebs»

Der Einsatz von Pflanzenextrakten bei der Behandlung von Prostataleiden hat eine lange Tradition. Phytosterole (pflanzliche, cholesterinähnliche Substanzen) sind hier die Hauptträger der entzündungshemmenden und zum Teil antiproliferativen Eigenschaften. Phytotherapeutische Präparate auf dieser Basis haben hier in den vergangenen 15 Jahren vor allem in den USA, aber auch in Europa eine weite Verbreitung gefunden (PC-SPES, Prostasol). Ihre Wirkungsweise ist jedoch oft in $\mathrm{Zu}-$ sammenhang mit der Beimengung von synthetischen Östrogenen gebracht worden. 
Seit 2008 sind an der AeskulapKlinik ca. 170 Prostatakarzinompatienten in nicht kurativem Stadium mit Sitosterol comp., einer Komposition von Diäthylstilböstrol $(1 \mathrm{mg} /$ Kapsel) und 8 phytosterolhaltigen Komponenten, behandelt worden. Nebenwirkungen dieser Therapie sind hauptsächlich östrogenbedingt (Magenbrennen, Wadenkrämpfe, Brustwarzenüberempfindlichkeit/-wachstum, Libidoverlust, Impotenz; selten: Herzschwäche, Thrombose, Lungenembolie). Nach bisheriger Teilauswertung führt Behandlung mit Sitosterol comp. in ca. 80\% der Fälle zu einem PSA-Abfall bzw. einer Stabilisierung des Wertes innerhalb der ersten 3 Monate (39/49 Patienten); ca. $60 \%$ profitieren von einer Langzeiteinnahme (>12 Monate, 33/52 Patienten). Die bisherigen Erfahrungen sind ermutigend, diese Präparation insbesondere

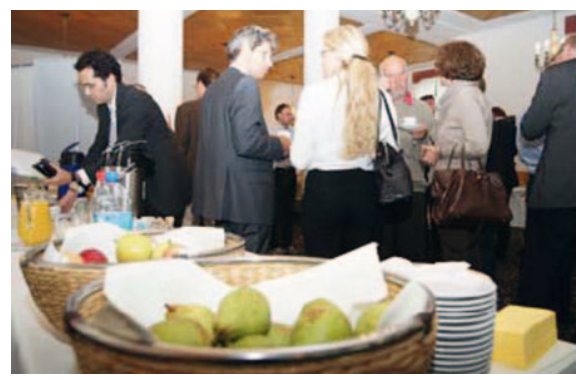

beim hormonresistenten Prostatakarzinom weiter einzusetzen und eine prospektive Studie vorzubereiten.

Prof. Dr. med. dent.

Christian E. Besimo:

«Tumorpatienten aus

oralmedizinischer Sicht»

Mögliche Zusammenhänge zwischen oralen und allgemeinen Erkrankungen sind in zunehmendem Masse Gegenstand wissenschaftlicher Untersuchungen. Ein intensiv untersuchtes Modell stellt dabei die marginale Parodontitis dar. So weisen zahlreiche Studien darauf hin, dass eine pathogenetische Beziehung zwischen marginaler Parodontitis und kardiovaskulären Erkrankungen besteht. Ähnlichkeiten der Pathogenese liegen auch mit der rheumatoiden Arthritis vor. Zudem ist eine wechselseitige Beeinflussung zwischen marginaler Parodontitis und Diabetes mellitus seit längerer Zeit bekannt. Zu den vom oralen Gesundheitszustand ebenfalls beeinflussten Krankheiten werden gerade bei Betagten Atemwegsinfektionen gezählt.

Auch eine Wechselwirkung zwischen Parodontitis und Malignomen wird diskutiert. So konnte in fünf von sechs Fallkontrollstudien ein signifikanter zwei- bis dreifacher Anstieg des Risikos für orale Malignome durch Parodontitis beobachtet werden. Ein erhöhtes Risiko wurde zudem für Malignome des oberen Gastrointestinaltrakts beschrieben, wobei die stärkste Korrelation zu Magenkarzinomen bestand. Die Situation für Lungen- und Pankreaskarzinome bleibt aufgrund der geringen Zahl von Studien und der kontroversen Datenlage unklar. Ein möglicher Zusammenhang zwischen Parodontitis und Gesamtmortalität von Malignomen wurde bisher lediglich in drei Kohortenstudien untersucht. Es konnte keine Korrelation nachgewiesen werden. Als mögliche pathogenetische Mechanismen wurden die Immunantwort auf parodontale Entzündung, eine Beeinträchtigung der Kontrolle des Zellwachstums, direkte Interaktion zwischen lokaler Immunantwort und Karzinogenese sowie eine erhöhte Produktion von Nitrosaminen durch orale Mikroorganismen diskutiert. Eine Mitberücksichtigung der oralen Gesundheit bei der Prävention und Therapie von oralen und oberen gastrointestinalen Malignomen erscheint aufgrund der Datenlage durchaus sinnvoll. 